\title{
Prediction of the itraconazole minimal inhibitory concentrations (MICs) of chromoblastomycosis agents using Fourier Transform-Infrared Spectroscopy and chemometrics
}

\author{
Daiane Heidrich 1,2; Valeriano Antonio Corbellini 3; Alessandra Koehler 1, \\ Maria Lúcia Scroferneker 1,2
}

1 Postgraduate Program in Medicine: Medical Sciences, Universidade Federal do Rio Grande do Sul. Rua Ramiro Barcelos, 2400 - CEP: 90035-003, Porto Alegre - RS, Brazil.

2 Department of Microbiology, Immunology and Parasitology, ICBS, Universidade Federal do Rio Grande do Sul. Rua Sarmento Leite, 500 - CEP 90050-170, Porto Alegre - RS, Brazil.

3 Department of Chemistry and Physics, Postgraduate Program in Health Promotion, Postgraduate Program in Environmental Technology, Universidade de Santa Cruz do Sul. Avenida Independência, 2293 - CEP 96815-900, Santa Cruz do Sul - RS, Brazil.

Objectives: use Fourier Transform-Infrared Spectroscopy (FTIR) and chemometrics to predict the itraconazole minimal inhibitory concentrations (MICs) for chromoblastomycosis (CBM) agents.

Methods: seventy-seven isolates of five genera of CBM agents were identified by comparison of ITS sequences of type strains available in GenBank using BLAST algorithm. Antifungal susceptibility of CBM agents to itraconazole (Sigma-Aldrich, USA) was performed according to the protocol M38-A2 of the Clinical and Laboratory Standards Institute (CLSI), utilizing the microdilution technique with a final antifungal concentration varying between $0.03-16 \mu \mathrm{g} / \mathrm{mL}$ to determine MICs. For FTIR analysis, the strains were prepared for Attenuated Total Reflection (ATR) with a new methodology using slices in glass fixing-modeling proposed. Five spectra were recorded from 4000 to $650 \mathrm{~cm}^{-1}$ for each strain. The data set was sample preprocessed by amplitude normalization and $1^{\text {st }}$ derivative $(5$ points) in Pirouette $\AA$ Software. PLS-FTIR algorithm was performed with quintuplicates using two orthogonal signal correction components and leave-one-out cross-validation.

Results: the strains were identified with $99 \%$ of identity with type strain of each species of thirteen species distributed on five genera of CBM. The identified species were: Fonsecaea pedrosoi (41), Fonsecaea monophora (15), Fonsecaea pugnacious (1), Fonsecaea nubica (1), Cladophialophora carrionii (3), Cladophialohora bantiana (1), Phialophora verrucosa (2), Phialophora americana (5), Exophiala spinifera (3), Exophiala xenobiotica (2), Rhinocladiella aquaspersa (1), Rhinocladiella tropicalis (1) e Rhinocladiella similis (1). The geometric mean of itraconazole MICs was $0.784 \mu \mathrm{g} / \mathrm{mL}$ ranging between $0.06 \mu \mathrm{g} / \mathrm{mL}$ and $32.0 \mu \mathrm{g} / \mathrm{mL}$, with MIC50 equal to 0.5 $\mu \mathrm{g} / \mathrm{mL}$ and MIC90 equal to $2.0 \mu \mathrm{g} / \mathrm{mL}$. FTIR analysis presented bands in the regions between 4000 and $3100 \mathrm{~cm}^{-1}$ (attributed to $\mathrm{NH}$ and $\mathrm{OH}$ stretching) together with the bands 1650 (amide I of proteins), 1050 and 1025 (polysaccharides bands) and the 900800 region (fingerprint) (Figure 1 ). In the PLS-FTIR model, the merit figures presented error less than 0.001 microgram $/ \mathrm{mL}$ and determination coefficient $\left(R^{2}\right)$ of 1.00 with 10 latent variables (Figure 2).

Sources: 屌CNPq
The major contributions to regression model were observed in fingerprint region (regression vector, Figure 1).

Conclusion: CBM is subcutaneous mycosis caused by different dematiaceous fungus belonging to many species and genera. Considering that each species can show different responses to antifungals, it is fundamental to determine the MIC. This model enables direct prediction of MICs, without necessity to identify the CBM agent or to perform antifungals susceptibility assays, which are more expensive and laborious than the methodology proposed here.

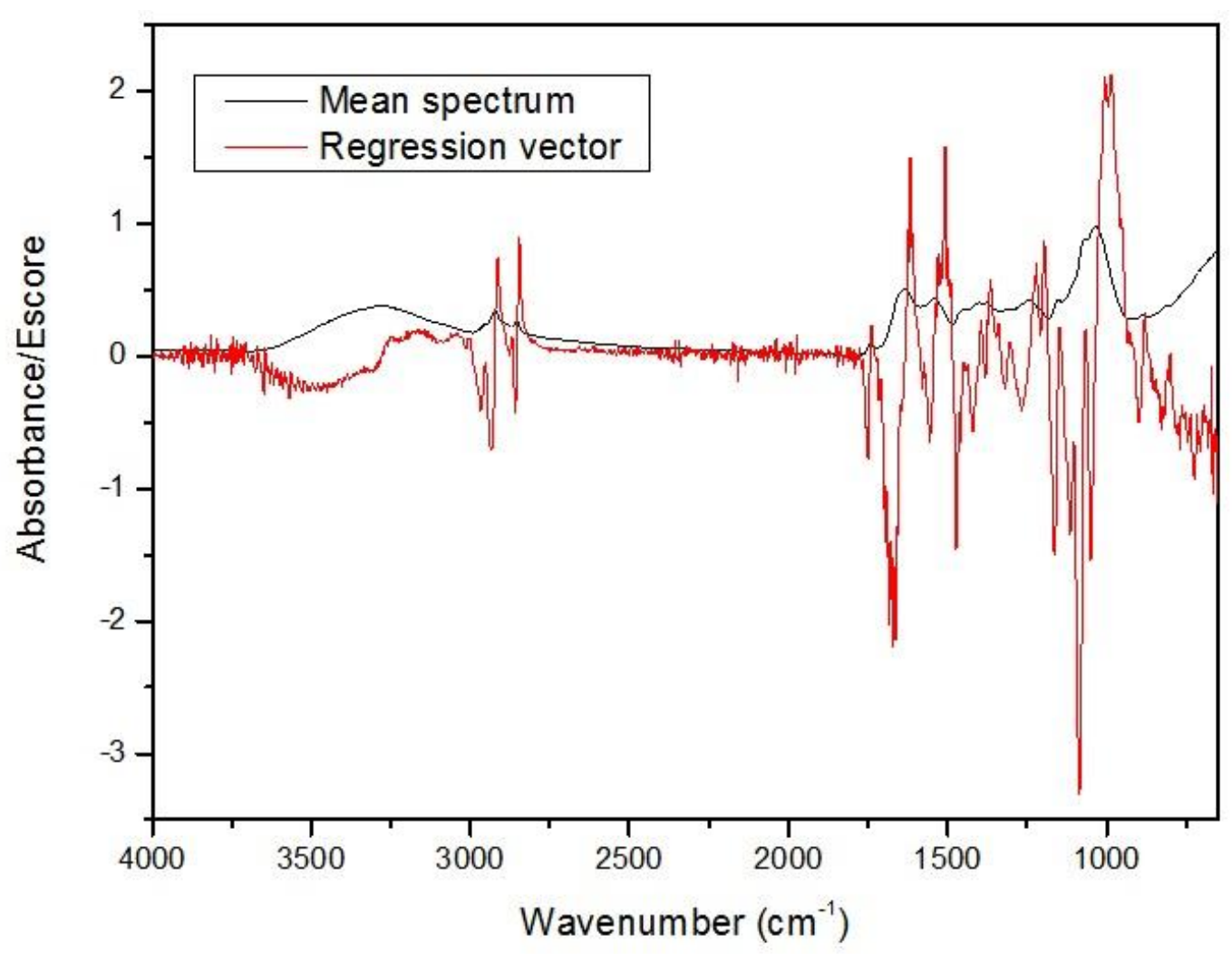

Figure 1. FT-IR mean spectrum of 77 samples (385 spectra) of chromoblastomycosis agents and regression vector of PLS-FTIR model of prediction of antifungal susceptibility to itraconazole supervised by CLSI method.

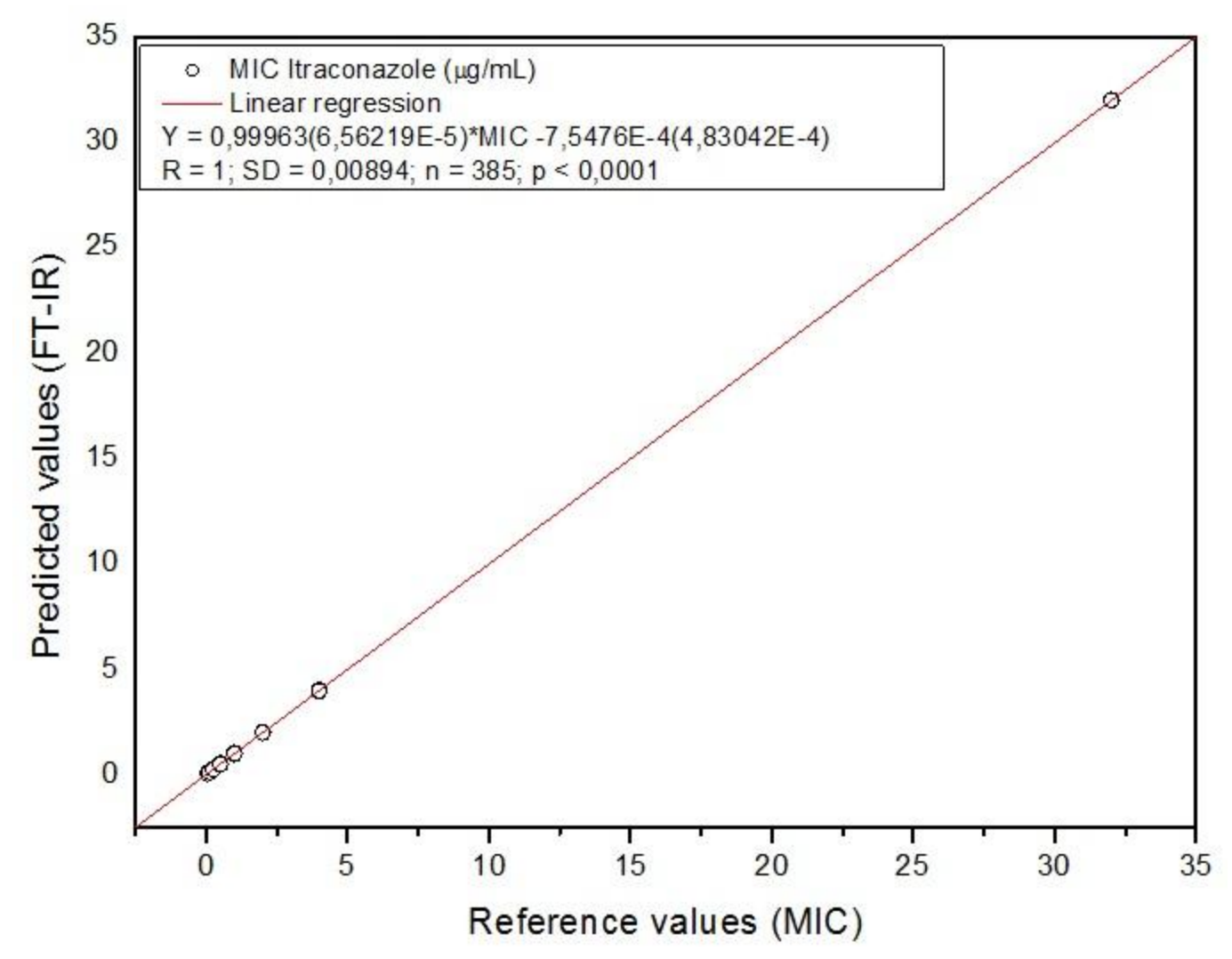

Figure 2. Regression analysis of PLS-FTIR model of prediction of MIC of 77 samples of chromoblastomycosis agents against itraconazole supervised by CLSI method.

srofern@ufrgs.br 